Ann. Biol. anim. bioch. Biophys., 1979, 19 (3 B), 749-755.

\title{
Hydrolyse enzymatique et stabilité en milieu acide des liaisons isopeptidiques de la L-méthionyl-caséine
}

\author{
par A. J. PUIGSERVER, L. C. SEN *, R. E. FEENEY *, J. R. WHITAKER * \\ Centre de Biochimie et de Biologie Moléculaire du CNRS \\ 31, chemin Joseph-Aiguier 13274 Marseille Cedex 2, France \\ * Department of Food Science and Technology, University of California, \\ Dovis, Colifornia 95615, USA.
}

Summary. Enzymatic hydrolysis and stability of the isopeptide bonds of methionyl-casein.

Active $\mathrm{N}$-hydroxysuccinimide esters of $\mathrm{N}$-acetyl-L-methionine and tert-butyloxycarbonyl derivatives of L-methionine and L-tryptophan were used to obtain covalent addition of the corresponding amino acids to casein through isopeptide bonds. These bonds were found to be more stable than peptide bonds in trifluoroacetic acid, under the conditions used for the unblocking of amino protecting group. In vitro hydrolysis studies performed with bovine and rat pancreatic juice indicated that digestibility of the modified casein derivatives was slightly lower than that of unmodified protein. The decreased in vitro digestibility is probably due to the steric hindrance of the added amino acids as well as to decrease in lysine residues available to trypsin in both pancreatic enzymes mixtures. Plasma amino acid levels for rats fed a 10 p. 100 protein diet of highly modified L-methionyl-casein or $\mathrm{N}$-acetyl-L-methionyl casein suggest that the isopeptide bond formed between the added amino acids and the $\varepsilon$-amino group of the lysyl residues of casein is readily hydrolyzed in vivo. Membrane-bound intestinal aminopeptidase was found to be quite efficient in hydrolyzing the isopeptide bond $\varepsilon-N-L-m e t h i o n y l-L-l y s i n e$.

\section{Introduction.}

La valeur nutritionnelle d'une protéine alimentaire est, dans une large mesure, le reflet direct de sa composition en acides aminés indispensables. Diverses méthodes ont été proposées pour augmenter la proportion de ces acides aminés dans les protéines et de tenter ainsi d'améliorer leur qualité. La teneur en méthionine ou en lysine de certaines protéines végétales importantes en raison de leurs nombreuses potentialités d'utilisation, ou de protéines animales a été améliorée grâce à l'utilisation de techniques chimiques (Bjarnason-Baumann, Pfaender ef Siebert, 1977 ; Puigserver ef al., 1978a), enzymatiques (Fujimaki, Arai et Yamashita, 1977) ou génétiques (Mertz, 1975). Si la supplémentation directe en acides aminés essentiels est l'opération la plus simple, et de ce fait la moins onéreuse, pour améliorer la valeur nutritionnelle d'une protéine alimentaire, elle n'est pas sans présenter des inconvénients. En effet, l'a ddition 
d'un acide aminé soufré, la méthionine, entraîne le plus souvent une modification importante des propriétés organoleptiques de l'aliment considéré. L'acide aminé ajouté peut aussi être à l'origine de réactions secondaires défavorables ou encore être perdu au cours des traitements technologiques. Enfin, il est important de souligner, que l'absorption intestinale d'un acide aminé libre ou au sein d'un peptide est réalisée par des mécanismes différents et qu'en outre, une corrélation entre hydrolyse peptidique et transport a été suggérée (Ugolev ef al., 1977).

Il était donc intéressant de savoir si la fixation covalente d'un acide aminé essentiel sur les résidus de lysine d'une protéine par une liaison isopeptidique pouvait éviter ces problèmes tout en augmentant la valeur nutritionnelle de la protéine étudiée. La stabilité en milieu acide et l'hydrolyse enzymatique de la liaison ainsi formée ont été étudiées sur la méthionyl-caséine et l'isodipeptide modèle $\varepsilon-\mathrm{N}$-méthionyllysine.

\section{Matériel et méthodes.}

Produits. - Les esters actifs (N-hydroxysuccinimide) des dérivés tert-butyloxycarbonyl de la L-méthionine et du L-tryptophane proviennent de la firme Bachem.

La N-acétyl-L-méthionine (Sigma) a été estérifiée au Laboratoire selon la méthode d'Anderson, Zimmerman et Gallahan (1964). La pepsine cristallisée de porc est un produit Worthington ; la caséine (Nutritional Biochemicals Corporation) a été utilisée sans autres purifications. Les sucs pancréatiques de porc et de rat nous ont été aimablement fournis par T. Corring (INRA, Jouy-en-Josas) et B. Schneeman (UCD, Californie). Le produit commercial "pancreatin 》 de bœuf de la firme Sigma a été considéré comme un bon équivalent, sur le plan enzymatique, du suc pancréatique de cette espèce.

L'isodipeptide radioactif $\left[{ }^{14} \mathrm{C}\right.$-méthyl]-\&-N-L-méthionyl-L-lysine (activité spécifique : $7 \mu \mathrm{Ci} / \mathrm{mmole}$ ) a été synthétisé (Puigserver et al., 1978b) en s'inspirant des méthodes classiques de synthèse peptidique.

Préparation des dérivés de la caséine. - La fixation covalente des L-amino acides activés sur les groupements $\varepsilon$-aminés des résidus de lysine de la caséine (liaison isopeptidique) a été réalisée à l'aide d'une technique décrite dans un précédent mémoire (Puigserver et al., 1978a). La caséine, en suspension à 2 p. 100 dans du tampon borate de sodium $0,1 \mathrm{M} \mathrm{à} \mathrm{pH} \mathrm{9,0,} \mathrm{est} \mathrm{incubée} \mathrm{pendant} 2 \mathrm{~h}$ à température ambiante en présence de l'acide aminé activé préalablement dissout dans du diméthyl-formamide (volume firal, 10 p. 100). Après hydrolyse des liaisons esters à pH 8,0 en présence d'hydroxylamine à la concentration finale $0,5 \mathrm{M}$ et élimination éventuelle du groupement protecteur tert-butyloxylcarbonyl par un traitement à l'acide trifluoroacétique anhydre, la protéine modifiée est dialysée contre de l'eau puis lyophilisée. La détermination de la teneur en tryptophane des protéines a été réalisée par la méthode d'Edelhoch (1967). Le taux de méthionine des échantillons de caséine commerciale ou chimiquement modifiée a été déterminé par analyse automatique après oxydation performique. La concentration des solutions protéiques a été estimée par la méthode au biuret.

Hydrolyse in vivo de la liaison isopeptidique. - L'expérimentation animale a été réalisée sur des groupes de 5 rats mâles de souche Sprague-Dawley (poids moyen: $75 \pm 6 \mathrm{~g}$ ). La composition chimique détaillée du régime protéique à $10 \mathrm{p} .100$ a déjà été 
décrite (Lee et al., 1978). Les animaux sont nourris ad libitum pendant 3 jours puis sacrifiés par décapitation et des échantillons de sang prélevés en présence d'EDTA.

Les plasmas, déprotéinisés par addition d'un égal volume d'acide sulfosalicylique à 6 p. 100 , sont conservés à $-15^{\circ} \mathrm{C}$ jusqu'au moment de l'analyse automatique, réalisée sur un appareil Beckman modèle $120 \mathrm{C}$.

Afin d'estimer l'hydrolyse in vivo de la liaison isopeptidique, nous avons comparé les concentrations plasmatiques de lysine chez des rats dont la seule composante protéique du régime est, soit de la caséine commerciale, soit une caséine hautement modifiée (>90 p. 100) par fixation covalente de méthionine ou de l'un de ses dérivés sur ses groupements aminés. En effet, si l'animal est incapable d'hydrolyser efficacement la liaison isopeptidique, la lysine de la caséine modifiée devient alors l'acide aminé limitant de cette protéine. Dans ce cas, le taux plasmatique de lysine libre, chez le rat ingérant une caséine dont une forte proportion des résidus lysyls a été modifiée, doit diminuer assez rapidement.

Hydrolyse in vitro des liaisons peptidiques ef isopeptidiques. - L'hydrolyse in vitro de la caséine ou de ses dérivés par les enzymes pancréatiques a été réalisée sur des solutions à 0,1 p. 100 de protéine dans un tampon phosphate monosodique $0,1 \mathrm{M}$ à $\mathrm{pH} 7,0$. Après une incubation de $48 \mathrm{~h}$ à $38^{\circ} \mathrm{C}$ en présence de quantités catalytiques d'enzymes $\left(1 / 100^{e}\right.$ en poids), les groupements aminés libres apparus par hydrolyse des liaisons peptidiques ou isopeptidiques sont quantifiés par la méthode de Fields (1972).

L'hydrolyse in vitro de l'isopeptide radioactif [ ${ }^{14} \mathrm{C}$-méthyl] $\varepsilon-\mathrm{N}$-L-méthionyl-Llysine a été suivie en séparant la méthionine libérée du peptide restant par chromatographie sur une colonne $(1 \times 6 \mathrm{~cm})$ de carboxyméthyl-Sephadex C 25 (Pharmacia) équilibrée avec du tampon phosphate de sodium $0,01 \mathrm{M}$ à pH 7,0. Dans ces conditions, la méthionine libre est éluée avec le volume mort de la colonne tandis qu'une force ionique élevée $(\mu=1)$ est nécessaire pour éluer l'isodipeptide. La quantité de méthionine libérée est estimée en mesurant la radioactivité à l'aide d'un compteur à scintillation liquide Tri-Carb modèle 2450.

\section{Résultats.}

Digestion in vitro de la méthionyl-caséine.

Pour aborder cette étude, il fallait en premier lieu démontrer que les conditions utilisées pour éliminer le groupement protecteur de la fonction amine des acides aminés fixés de manière covalente sur les résidus de lysine n'entraînent aucune hydrolyse de la protéine. L'utilisation de la tert-butyloxycarbonyltryptophyl-caséine comme modèle de protéine chimiquement modifiée, permet d'évaluer l'hydrolyse des liaisons peptidiques par dosage des groupements aminés libres apparus et celle des liaisons isopeptidiques en suivant la diminution d'absorption à $280 \mathrm{~nm}$ de la solution protéique dialysée.

Les liaisons peptidiques de la caséine non traitée ou de ses dérivés ne commencent à être hydrolysées qu'à $50^{\circ} \mathrm{C}$ en présence d'acide trifluoroacétique à 75 p. 100 (fig. 1). Cette température élevée et l'excès molaire important d'acide par rapport à la concentration des liaisons isopeptidiques ( 600 fois) ne sont généralement pas utilisés pour 
éliminer le groupement fert-butyloxycarbonyl. Dans des conditions où l'hydrolyse peptidique se produit, la liaison isopeptidique est encore résistante. Dans le cas de la tryptophyl-caséine, la liaison isopeptidique est donc plus stable en milieu acide que les liaisons peptidiques.
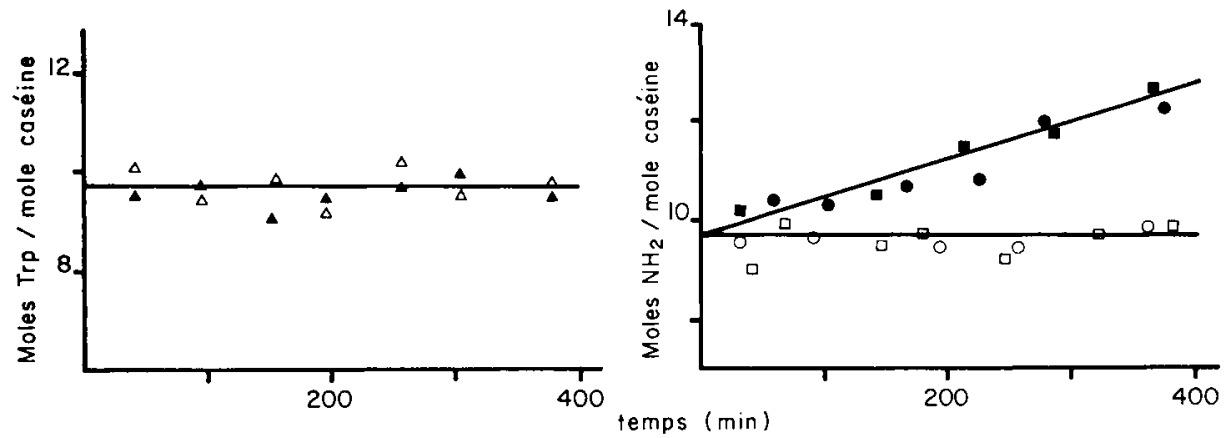

FIG. 1. - Stabilité des liaisons peptidiques et isopeptidiques dans l'acide trifluoroacéfique à $50^{\circ} \mathrm{C}$. La caséine témoin $(25 \mathrm{mg} / \mathrm{ml})$, est incubée dans de l'acide pur $(O)$ ou à 75 p. $100(\bullet)$. Mêmes conditions pour la méthionyl-caséine $(\square,-$,$) et la tryptophyl-caséine (\Delta, \mathbf{\Delta})$. Dans ce dernier cas, la teneur en tryptophane est déterminée après dialyse de chaque prélèvement contre de l'eau.

L'hydrolyse in vitro des dérivés de la caséine (méthionyl- et $\mathrm{N}$-acétyl-méthionyl-) par les enzymes de la sécrétion exocrine du pancréas de bœuf ou de rat est moins poussée que celle de la caséine témoin (tabl. 1). On peut aussi remarquer que la charge du résidu ajouté est importante car le dérivé $N$-acétylé est moins bien hydrolysé que la méthionyl-caséine. La fixation covalente d'un acide aminé sur les groupements $\varepsilon$ aminés d'une protéine alimentaire pourrait donc affecter sa digestibilité in vivo. A ce stade de l'expérience, on ne peut savoir si l'augmentation du nombre des groupements aminés libres résulte uniquement de l'hydrolyse des liaisons peptidiques ou si l'hydrolyse de la liaison isopeptidique intervient également.

\section{TABLEAU 1}

Hydrolyse enzymatique in vitro des dérivés de la caséine

\begin{tabular}{|c|c|c|c|}
\hline \multirow{2}{*}{ Protéine } & \multirow{2}{*}{$\begin{array}{l}\text { Degré de modification } \\
\text { (p. 100) }\end{array}$} & \multicolumn{2}{|c|}{$\begin{array}{l}\text { Hydrolyse relative (p. } 100 \text { ) } \\
\text { par des sucs pancréatiques : }\end{array}$} \\
\hline & & Rat & Bœuf \\
\hline $\begin{array}{l}\text { Caséine témoin . . . . . . . } \ldots \ldots \ldots \ldots \\
\text { Méthionyl-caséine } \ldots \ldots \ldots \ldots \ldots \\
\text { N-acétyl-méthionyl-caséine } \ldots \ldots\end{array}$ & $\begin{array}{r}0 \\
59 \\
57\end{array}$ & $\begin{array}{r}100 \\
95 \\
71\end{array}$ & $\begin{array}{r}100 \\
90 \\
84\end{array}$ \\
\hline
\end{tabular}

Le degré de modification des dérivés de la caséine a été établi en estimant la teneur en groupements aminés libres avant et après élimination du groupement tert-butyloxycarbonyl. Ce résultat a été confirmé par la détermination des compositions en acides aminés des protéines. 
Hydrolyse de la liaison isopeptidique.

Les niveaux de lysine et de méthionine libres dans le plasma des rats alimentés en rations protéiques contenant de la caséine non traitée ou ses dérivés, sont indiqués dans le tableau 2.

TABLEAU 2

Niveaux de deux acides aminés plasmatiques chez des rats nourris à l'aide de protéines modifiées

\begin{tabular}{|c|c|c|}
\hline \multirow{2}{*}{ Protéines } & \multicolumn{2}{|c|}{$\mu$ moles $/ 100 \mathrm{ml}$ plasma } \\
\hline & Lysine & Méthionine \\
\hline $\begin{array}{l}\text { Caséine témoin } \ldots \ldots \ldots \ldots \ldots \ldots \\
\text { Boc-méthionyl-caséine } \ldots \ldots \ldots \ldots \ldots \\
\text { Méthionyl-caséine } \ldots \ldots \ldots \ldots \ldots \\
\text { N-acétyl-méthionyl-caséine } \ldots \ldots \ldots\end{array}$ & $\begin{array}{r}60.5 \pm 8.3 \\
5.7 \pm 1.4 \\
72.4 \pm 4.8 \\
34.5 \pm 6.5\end{array}$ & $\begin{array}{r}4.1 \pm 1.2 \\
4.0 \pm 1.0 \\
39.3 \pm 3.5 \\
4.5 \pm 1.7\end{array}$ \\
\hline
\end{tabular}

Les dérivés de la caséine sont modifiés à plus de 90 p. 100.

Boc $=$ = tert-butyloxycarbonyl.

La liaison isopeptidique de la méthionyl-caséine et de la $\mathrm{N}$-acétyl-méthionylcaséine semble avoir été hydrolysée avec la même efficacité. Par contre, la présence du groupement protecteur tert-butyloxycarbonyl est incompotible avec la libération de l'acide aminé ajouté. Bien que le temps d'expérience soif court, il est suffisant pour montrer que les résidus de lysine de la tert-butyloxycarbonyl-méthionyl-caséine ne peuvent probablement pas être libérés de l'acide aminé ajouté. Dans ce cas, il en résulte une diminution importante de la concentration plasmatique de lysine. Ce type d'observation a déjà été décrit dans le cas de caséines $\varepsilon$-alkylées (Lee et al., 1978) don† on sait bien que l'alkyl-lysine ne peut être utilisée directement par l'animal pour sa biosynthèse protéique.

Les résultats ci-dessus pourraient indiquer que l'activité enzymatique responsable de l'hydrolyse de la liaison isopeptidique est de type aminopeptidasique. Cette hypothèse est confirmée par l'expérience dont les résultats figurent dans le tableau 3.

TABLEAU 3

Hydrolyse de l'isodipeptide $\left[{ }^{14} \mathrm{C}\right.$-méthyl] $\varepsilon-\mathrm{N}$-L-méthionyl-L-lysine par les enzymes du tractus digestif

\begin{tabular}{|c|c|c|c|}
\hline & Enzymes & $\begin{array}{l}\text { Hydrolyse du substrat } \\
\text { (p. 100) }\end{array}$ & $E / S$ poids/poids \\
\hline Pore & 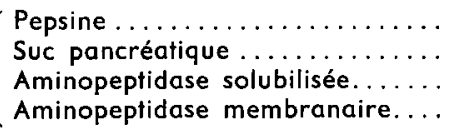 & $\begin{array}{r}2 \\
3 \\
95 \\
91\end{array}$ & $\begin{array}{l}1: 1 \\
1: 6 \\
1: 500 \\
1: 500\end{array}$ \\
\hline Rat & Aminopeptidase membranaire.... & 93 & $1: 500$ \\
\hline
\end{tabular}

Un $\mathrm{ml}$ d'une solution de substrat $5 \mathrm{mM}$ dans du tampon phosphate monosodique $50 \mathrm{mM}$ à pH 7,0 est incubé en présence d'enzymes pendant $3 \mathrm{~h}$ à $37^{\circ} \mathrm{C}$. 
L'aminopeptidase de l'intestin grêle de rat ou de porc est capable de catalyser efficacement l'hydrolyse de la liaison isopeptidique. De plus, l'enzyme solubilisée ou encore au sein de la membrane de la bordure en brosse des entérocytes (Maroux, Louvard et Baratti, 1973) hydrolyse ce substrat avec des vitesses comparables. II est important de noter que les protéases des sucs pancréatique et gastrique sont incapables d'hydrolyser l'isodipeptide.

\section{Discussion et conclusion.}

La fixation covalente de la méthionine ou de son dérivé $\mathrm{N}$-acétylé sur les résidus de lysine de la caséine se traduit, in vitro, par une hydrolyse moins poussée de la protéine modifiée par les enzymes pancréatiques. Ceci résulte probablement d'une moins bonne accessibilité des liaisons hydrolysables, dans les protéines modifiées par rapport à la protéine témoin, ef de l'impossibilité pour la trypsine de cliver les liaisons de la lysine. Il est important de conserver à cette expérience son caractère d'estimation indirecte de la possibilité pour un animal d'utiliser une protéine chimiquement modifiée par fixation covalente d'acides aminés essentiels.

Les enzymes pancréatiques et la pepsine ne sont pas capables d'hydrolyser la liaison isopeptidique $\varepsilon-\mathrm{N}$-L-méthionyl-L-lysine car la plupart d'entre elles sont des endopeptidases. Cependant, elles ne semblent pas davantage aptes à cliver cette liaison lorsque le groupement $\alpha$-aminé de la méthionine est acétylé. Cela tient probablement à la géométrie du site de spécificité de ces enzymes qui nécessite la présence d'un squelette peptidique normal pour agir efficacement.

L'hydrolyse in vivo de la liaison isopeptidique de la méthionyl- et de la $\mathrm{N}$-acétylméthionyl-caséine est particulièrement intéressante. Si l'aminopeptidase intestinale est la seule enzyme réalisant cette réaction, on doit alors supposer que la déacylation de la $\mathrm{N}$-acétyl-méthionyl-lysine précède l'hydrolyse de la liaison isopeptidique. Enfin, il est important de remarquer que l'hydrolyse de cette liaison qui résulte d'une modification chimique de la caséine est réalisée par une enzyme constitutive de la membrane de la bordure en brosse des entérocyłes. Ceci pourrait expliquer le fait qu'un acide aminé indispensable fixé par liaison isopeptidique sur une protéine alimentaire semble être normalement utilisé chez le rat (Puigserver et al., 1978a).

Il faut enfin souligner que l'expérimentation sur rats, felle que nous l'avons conduite, ne permet pas une mesure quantitative des possibilités réelles d'utilisation de la méthionine ajoutée. En particulier, il est difficile de savoir si le taux élevé de méthionine libre dans le sang des animaux ayant ingéré de la méthionyl-caséine hautement modifiée ( 0,95 p. 100 de méthionine dans le régime), résulte de la quantité anormalement élevée de cet amino-acide dans l'alimentation ou traduit une difficulté d'utilisation.

Commission CNERNA Digestion-Absorption/Associotion des Physiologistes, Paris 5-6 octobre 1978.

Remerciements. - Nous tenons à exprimer toute notre gratitude à $D$. Gratecos pour la préparation de l'aminopeptidase solubilisée ef membranaire de porc et de rat et à D. Filippi pour sa précieuse collaboration à l'éfude de l'hydrolyse de la liaison isopeptidique. Nous remercions aussi A. J. Clifford (Université de Californie, Davis) de son aide pour l'expérimentation animale. 


\section{Références}

ANDERSON G. W., ZIMMERMAN J. E., CALLAHAN F. M., 1964. The use of esters of N-hydroxysuccinimide in peptide synthesis. J. am. chem. Soc., 86, 1839-1842.

BJARNASON-BAUMANN B., PFAENDER P., SIEBERT G., 1977. Enhancement of the biological value of whey protein by covalent addition into peptide linkage of limiting essential amino acids. Nutr. Metab., 21, Suppl. 1, 170-171.

EDELHOCH H., 1967. Spectroscopic defermination of tryptophan and tyrosine in proteins. Biochemistry, 6, 1948-1954.

FIELDS R., 1972. The rapid determination of amino groups with TNBS. Meth. Enzym., 25B, 464-468.

FUJIMAKI M., ARAI S., YAMASHITA M., 1977. Enzymatic protein degradation and resynthesis for protein improvement. Adv. Chem. Series, 160, 156-184.

LEE H. S., SEN L. C., CLIFFORD A. J., WHITAKER J. R., FEENEY R. E., 1978. Effect of reductive alkylation of the $\varepsilon$-amino group of lysyl residues of casein on its nutritive value in rats. $J$. Nutr., 108, 687-697.

MAROUX S., LOUVARD D., BARATTI J., 1973. The aminopeptidase from hog intestinal brush border. Biochim. biophys. Acta, 321, 282-295.

MERTZ E. T., 1975. Breeding for improved nutritional values in cereals, 1-12. In FRIEDMAN M., Protein nutritional quality of foods, and feeds (vol. 1, partie 2. Marcel Dekker. Inc. New York).

PUIGSERVER A. J., SEN L. C., CLIFFORD A. J., FEENEY R. E., WHITAKER J. R., 1978a. A method for improving the nutritional value of food proteins : Covalent attachment of amino acids. Adv. exp. Med. Biol., 105, 587-612.

PUIGSERVER A. J., SEN L. C., CLIFFORD A. J., FEENEY R. E., WHITAKER J. R., 1978b. Biovailability of methionine and $\mathrm{N}$-acetyl-methionine covolently linked to casein. J. Nutr. (soumis pour publication).

UGOLEV A. M., TIMOFEEVA N. M., SMIRNOVA L. F., DE LAEY P., GRUZDKOV A. A., IEZUITOVA N. N., MITYUSHOVA N. M., ROSHCHINA G. M., GURMAN E. G., GUSEN V. M., TSVETKOVA V. A., SHCHERBAKOV G. G., 1977. Membrane and intracellular hydrolysis of peptides : differentiation, role and interrelations with transport. In Peptide transport and hydrolysis, Ciba Found. Symp., 50, 221-243, Assoc. Sci. Publ., Amsterdam. 